\title{
CONHECIMENTO DE ACADÊMICOS DE ENFERMAGEM SOBRE OS CUIDADOS DO ENFERMEIRO AO PACIENTE EM RECUPERAÇÃO ANESTÉSICA
}

\author{
Knowledge of nursing students about nursing care for patients recovering from anesthesia
}

\author{
Conocimiento de estudiantes de enfermería sobre la atención \\ de enfermeras para pacientes sometidos a recuperación anestésica
}

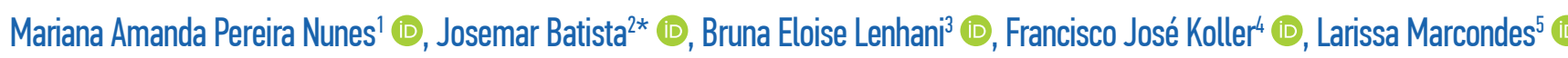

RESUMO: Objetivo: Identificar o conhecimento de acadêmicos de enfermagem relacionado aos cuidados e às ações desenvolvidas pelo enfermeiro em sala de recuperação pós-anestésica com vistas à segurança do paciente. Método: Estudo descritivo-exploratório, de abordagem qualitativa, realizado com 30 acadêmicos de enfermagem de uma instituição privada de ensino superior da Região Sul do Brasil. A coleta de dados ocorreu no mês de outubro de 2018, mediante aplicação de questionário semiestruturado. Empregou-se a análise de conteúdo de Bardin para tratamento dos dados. Resultados: Com base nos depoimentos dos entrevistados, emergiu a seguinte categoria: Cuidados e ações do enfermeiro para segurança do paciente, em sala de recuperação pós-anestésica, percebidos pelos acadêmicos de enfermagem. Cuidados rotineiros e mecanicistas, comunicação, liderança, aplicação do processo de enfermagem e uso de protocolos e escalas foram reconhecidos pelos acadêmicos como fundamentais para a segurança do paciente na recuperação. Conclusão: Os acadêmicos de enfermagem reconheceram elementos promotores do cuidado seguro relacionados à assistência de enfermagem na recuperação anestésica.

Palavras-chave: Educação em enfermagem. Cuidados de enfermagem. Enfermeiras e enfermeiros. Período de recuperação da anestesia. Segurança do paciente.

ABSTRACT: Objective: To identify the knowledge of nursing students about nursing care and actions in the post-anesthesia care unit regarding patient safety. Method: This is an exploratory descriptive study with a qualitative approach, conducted with 30 nursing students from a private higher education institution in Southern Brazil. Data were collected by administering a semi-structured questionnaire in October 2018. We adopted Bardin's content analysis for data processing. Results: The following category emerged based on the interviewees' statements: Nursing care and actions in the post-anesthesia care unit regarding patient safety, perceived by nursing students. The students acknowledge routine and mechanistic care, communication, leadership, nursing process application, and use of protocols and scales as fundamental for patient safety when recovering. Conclusion: Nursing students identified safe care elements related to nursing care in anesthesia recovery.

Keywords: Education, nursing. Nursing care. Nurses. Anesthesia recovery period. Patient safety.

RESUMEN: Objetivo: identificar el conocimiento de los estudiantes de enfermería relacionados con la atención y las acciones desarrolladas por las enfermeras en la sala de recuperación postanestésica, con miras a la seguridad del paciente. Método: Este es un estudio descriptivo y exploratorio con un enfoque cualitativo, realizado con 30 estudiantes de enfermería de una institución privada de educación superior en el sur de Brasil. La recopilación de datos tuvo lugar en octubre de 2018, mediante la aplicación de un cuestionario semiestructurado. El análisis de contenido temático de Bardin se utilizó para el procesamiento de datos. Resultados: surgió la siguiente categoría: Atención de enfermería y acciones para la seguridad del paciente en una sala

'Enfermeira; Graduada em Bacharelado em Enfermagem pelas Faculdades Integradas Santa Cruz de Curitiba (FARESC) - Curitiba (PR), Brasil.

${ }^{2}$ Enfermeiro; mestre em Enfermagem pela Universidade Federal do Paraná (UFPR). Professor adjunto da FARESC - Curitiba (PR), Brasil.

${ }^{3}$ Enfermeira; doutoranda em Enfermagem pela UFPR. Professora adjunta da FARESC - Curitiba (PR), Brasil.

"Enfermeiro; mestre em Enfermagem pela UFPR - Curitiba (PR), Brasil. Professor adjunto da FARESC - Curitiba (PR), Brasil.

${ }^{5}$ Enfermeira; mestre em Enfermagem pela UFPR. Professora adjunta da FARESC - Curitiba (PR), Brasil.

*Autor correspondente: josemar.batista@hotmail.com

Recebido: 28/03/2019 - Aprovado: 15/10/2019

DOI: $10.5327 / Z 1414-4425201900040009$ 
de recuperación postanestésica, según lo perciben los estudiantes de enfermería. Los cuidados académicos y de rutina, la comunicación, el liderazgo, la aplicación del proceso de enfermería y el uso de protocolos y escalas fueron reconocidos por los académicos como críticos para la seguridad del paciente en la recuperación. Conclusión: los estudiantes de enfermería reconocieron elementos que promueven la atención segura, relacionados con la atención de enfermería en la recuperación anestésica.

Palabras-clave: Educación en enfermería. Atención de enfermería. Enfermeras y enfermeros. Periodo de recuperación de la anestesia. Seguridad del paciente.

\section{INTRODUÇÃO}

O programa Cirurgias Seguras Salvam Vidas, lançado pela Organização Mundial da Saúde (OMS), em seu segundo desafio global para segurança do paciente, visa a reforçar práticas cirúrgicas seguras cujo objetivo é a redução de atos inseguros, incluindo os relacionados à sala de recuperação pós-anestésica (SRPA) ${ }^{1}$.

A SRPA é o local destinado a receber pacientes após o ato cirúrgico-anestésico. Preocupações com segurança, nesse ambiente, incluem questões ligadas a sobrecarga de trabalho, identificação, avaliação e transferências de pacientes, bem como uso de equipamentos e analgésicos que potencializam riscos assistenciais e demandam ações com vistas a prevenir incidentes por parte dos profissionais de enfermagem ${ }^{2}$.

Ante a ocorrência de falhas técnicas e/ ou de habilidades não técnicas que contribuem para a ocorrência de eventos adversos em anestesia ${ }^{3}$, associados a alterações sistêmicas do paciente no pós-operatório, as quais o predispõe a complicações de origem circulatória, respiratória e gastrointestinal $^{4}$, torna-se necessário à equipe de saúde, especialmente à enfermagem, precisão na avaliação clínica, a fim de identificar alterações hemodinâmicas e favorecer a qualidade e a segurança assistencial'5.

Estudo conduzido com relatórios de incidentes em hospitais dos Estados Unidos identificou que, na análise de causa raiz de 36 eventos adversos, os erros foram causados por falhas de padronização e/ou política em processos para a administração segura de anestésicos ${ }^{3}$. Esses fatores podem potencializar a ocorrência de complicações, como: hipotermia, hipoxemia, apneia, tremores, náuseas, vômitos, disritmias cardíacas e retenção urinária. Da mesma forma, podem elevar o grau de dependência de cuidados ${ }^{6}$, que, se não gerenciados, predispõem a eventos adversos graves e preveníveis.

A recuperação segura do paciente, no período pós-operatório na SRPA, depende do uso de recursos e equipamentos de monitoração associados aos cuidados de enfermagem, os quais devem ser fundamentados em conhecimentos científicos e habilidades técnicas para a realização de intervenções seguras, que envolvem o processo cirúrgico e anestésico ${ }^{4}$. Cabe destacar ainda que, para a efetiva implementação da sistematização da assistência de enfermagem perioperatória (SAEP) como instrumento metodológico para assistir ao paciente de formal integral, contínua e segura, faz-se necessário que o enfermeiro tenha conhecimento sobre a aplicabilidade dos diagnósticos de enfermagem, suas intervenções e resultados esperados ${ }^{7}$.

Portanto, investigar o conhecimento dos acadêmicos de enfermagem referente à assistência que deve ser prestada pelo enfermeiro ao paciente no decorrer da recuperação anestésica serve como ferramenta para execução de melhorias futuras no processo de ensino-aprendizagem, com reflexo na prática assistencial. Diante do exposto, tem-se como questão norteadora: qual é o conhecimento dos acadêmicos de enfermagem em relação aos cuidados e às ações desempenhadas pelo enfermeiro na SRPA?

\section{OBJETIVO}

Identificar o conhecimento dos acadêmicos de enfermagem relacionado aos cuidados e às ações desenvolvidas pelo enfermeiro na SRPA com vistas à segurança do paciente.

\section{MÉTODO}

Trata-se de um estudo descritivo-exploratório, de abordagem qualitativa, realizado com acadêmicos do curso de graduação em enfermagem de uma instituição de ensino superior privada da Região Sul do Brasil. A população-alvo foi composta de 68 acadêmicos, aprovados na disciplina teórica Centro Cirúrgico, Central de Materiais e SRPA, ofertada no $3^{\circ}$ ano do curso $\left(5^{\circ}\right.$ período).

Elencou-se para critérios de inclusão: ser acadêmico do ensino superior de enfermagem; estar devidamente matriculado (cursando o $6^{\circ}$, o $7^{\circ}$ ou o $8^{\circ}$ período); e ter idade igual ou superior a 18 anos. Foram excluídos os questionários 
devolvidos em branco. Após o convite individual e os esclarecimentos dos objetivos do estudo, 30 acadêmicos aceitaram participar da pesquisa. Não houve nenhuma desistência e/ou exclusão. Foram entregues aos acadêmicos o questionário e o termo de consentimento livre e esclarecido (TCLE), os quais foram devolvidos aos pesquisadores.

Os dados foram coletados em outubro de 2018, mediante aplicação de questionário semiestruturado, elaborado pelos pesquisadores, composto de questões que visavam a identificar os dados sociodemográficos e o conhecimento dos acadêmicos em relação ao cuidado a ser prestado na SRPA:

1. O que você entende por SRPA?

2. Qual é a importância do enfermeiro na SRPA?

3. Quais são os principais cuidados que você considera necessários aos pacientes no pós-operatório imediato na SRPA?

4. Como você percebe que o cuidado de enfermagem na SRPA auxilia na segurança do paciente cirúrgico?

Para análise dos dados, foi empregada a análise de conteúdo do tipo temática ${ }^{8}$. Na fase de pré-análise, foram realizadas leituras aprofundadas dos depoimentos com a finalidade de identificar as ideias centrais. A exploração do material empírico ocorreu com a intenção de sintetizar as informações e, assim, compor as categorias iniciais e intermediárias. Essas categorias foram agrupadas tematicamente, de modo a originar uma única categoria final e conceber a interpretação do objeto de estudo.

Para a garantia do anonimato, os participantes foram identificados pela letra $\mathrm{A}$ seguida de numeral relativo à ordem de devolutiva dos questionários (A1, A2, ..., A30). Este estudo foi aprovado pelo Comitê de Ética em Pesquisa da instituição proponente, sob o número da Certificado de Apresentação para Apreciação Ética (CAAE) 95955118.3.0000.5529 e parecer $\mathrm{n}^{\circ}$ 2.961.674. Atendeu aos preceitos éticos e legais da Resolução no 466/2012, do Conselho Nacional de Saúde.

\section{RESULTADOS}

Dos 30 acadêmicos participantes, 26 eram do sexo feminino. A média de idade foi de 28 anos, com variação da faixa etária entre 19 e 43 anos. Participaram 12 acadêmicos do $6^{\circ}$ período, dois do $7^{\circ}$ e 16 do $8^{\circ}$ período. Com base nos dados extraídos, foi possível elencar a seguinte categoria temática: Cuidados e ações do enfermeiro para segurança do paciente em SRPA percebidos pelos acadêmicos de enfermagem.
Os acadêmicos de enfermagem externaram que a assistência de enfermagem na SRPA se compõe de cuidados técnicos e rotineiros como, por exemplo, aferir sinais vitais e avaliar intensidade da dor e nível neurológico. Também consideraram medidas de conforto, avaliação de curativos e de dispositivos utilizados em procedimentos intervencionistas e cirúrgicos, visando a controlar as funções fisiológicas. Esses cuidados são exemplificados pelos seguintes discursos:

"Controle dos sinais vitais, manter o paciente aquecido, verificar e anotar a intensidade da dor, verificar sinais de hipóxia, esforço respiratório e/ou cianose" (A30).

"Avaliar nível de consciência, atividade motora, avaliar ocorrência de sangramento, atentar-se às queixas do paciente, oferecendo medidas de conforto" (A10).

"Ver o curativo das cirurgias, ver se não tem hemorragias, avaliar a dor quanto à queixa do paciente, [...] ver todos os dispositivos e seus devidos cuidados, como sonda nasoenteral, sonda vesical de demora, dreno de suctor, tórax, entre outros" (A19).

"Sempre orientar o paciente quanto ao tempo e espaço devido à anestesia e atentar para que o paciente não broncoaspire" (A28).

"Manter monitoramento contínuo, como saturação, pressão arterial, temperatura, frequência cardíaca” (A27).

O cuidado mecanicista, segundo os acadêmicos, deve ocorrer com a integração do conhecimento teórico-prático articulado à utilização de protocolos e escalas padronizados. Os participantes ainda salientaram a importância de identificar complicações agudas, as quais necessitam de intervenções imediatas pela equipe de saúde:

\footnotetext{
"Monitorar os sinais vitais conforme os protocolos, além dos sinais vitais e sintomas que o paciente refere ou aparenta, como dor ou cianose. Troca de curativos, se necessário, hidratação e medicação conforme prescrição médica" (A8).

"Realizar o monitoramento de sinais vitais como pressão arterial, frequência cardíaca, saturação de
} 
oxigênio, aferindo a cada 15 minutos junto à escala de Aldrete e Kroulik” (A10).

"É de extrema importância o conhecimento técnico e teórico da equipe de enfermagem para que possa identificar sinais e sintomas de possível complicação, como, por exemplo, choque hipovolêmico. [...] O enfermeiro deve estar ali atento a qualquer alteração, visando reconhecer o risco de queda, risco de agitação, reação alérgica a medicamentos ou anestesia” (A9).

Nessa perspectiva, os acadêmicos ressaltaram diferentes atividades desempenhadas pelo enfermeiro em sua prática profissional na SRPA. Citaram a comunicação interpessoal com os pacientes e a equipe de trabalho e o exercício profissional baseado em competências como elementos relevantes para que ocorra a recuperação segura do paciente:

"O enfermeiro tem que ficar atento quanto à recuperação do paciente e ser comunicativo com sua equipe" (A16).

"Avaliação do risco de infecção, [...] passar ao paciente as informações necessárias para a melhor recuperação do mesmo.” (A24)

"O enfermeiro se torna essencial, pois possui conhecimento científico para agir em emergências, e a liderança no setor faz com que o cuidado prestado seja de qualidade” (A8).

"Enquanto não é papel do médico gerenciar a SRPA, o enfermeiro é o profissional mais capacitado para tanto" (A7).

"Saber se tem leitos disponíveis para os pacientes que realizaram cirurgias e serão liberados em horários diferentes e que vão permanecer na SRPA por períodos diferentes. É responsável pelo dimensionamento dos funcionários e manter os estoques de materiais necessários” (A1).

Paralelamente a isso, os acadêmicos assinalaram, de maneira positiva, a implementação do processo de enfermagem com vistas a garantir a segurança do paciente e a qualidade do cuidado dispensado pelo enfermeiro em SRPA.
"O enfermeiro tem o papel de cuidar/prescrever e orientar sua equipe sobre procedimentos e cuidados necessários dos pacientes” (A18).

"A realização do exame físico e [sistematização da assistência de enfermagem] SAE” (A21).

\section{DISCUSSÃO}

A SRPA exige da equipe de saúde, especialmente do enfermeiro, desempenhar ações e cuidados específicos ante a complexidade e a dinamicidade de fatores que potencializam a (in) segurança do paciente nessa fase cirúrgica ${ }^{2}$. Os acadêmicos de enfermagem apontam para a importância de procedimentos e ações rotineiras como primordiais para o cuidado seguro do paciente. Esse dado corrobora o relato de experiência de uma acadêmica de enfermagem a respeito das atividades desenvolvidas em uma SRPA de um hospital universitário do Rio Grande do Sul ${ }^{9}$. Outro estudo realizado com profissionais de enfermagem atuantes em SRPA de um hospital privado do oeste do estado do Paraná trouxe em seus relatos a avaliação de sinais vitais, dor e cuidados específicos com a ferida cirúrgica visando à identificação precoce de possíveis eventos adversos 5 .

O referido estudo paranaense evidenciou que a equipe de enfermagem se detém, principalmente, à avaliação dos sistemas respiratório, circulatório e neurológico, por meio de avaliação do nível de consciência ${ }^{5}$, confirmando os achados da presente pesquisa. Os cuidados concebidos pelos acadêmicos de enfermagem visam ao atendimento sistemático e integral, estando atentos a possíveis complicações oriundas do processo anestésico-cirúrgico. Dessa forma, contribuem para a oferta do cuidado no pós-operatório com mais segurança.

Os discentes afirmaram associar aos cuidados tecnicistas o uso de escalas e protocolos cujo objetivo é sistematizar o cuidado ao paciente para a prevenção de potenciais incidentes que sustentem as ações a serem desempenhadas pelos profissionais. Sabe-se que a precisão na mensuração dos sinais vitais e na avaliação pelo profissional enfermeiro, a fim de identificar sinais e sintomas de complicações decorrentes do procedimento anestésico-cirúrgico, é determinante para condutas assertivas. A escala de Aldrete e Kroulik, citada pelos acadêmicos, é um instrumento de fácil aplicação e difundido nas SRPA cujo propósito é avaliar a evolução dos pacientes no período pós-anestésico por meio da análise da atividade 
muscular, da respiração, da circulação, da consciência e da saturação de oxigênio ${ }^{10}$.

Por essa ótica, uma investigação conduzida com oito técnicos e um enfermeiro atuantes em SRPA de hospital de médio porte do Sul do Brasil apontou a utilização dessa escala como forma de avaliação do paciente cirúrgico na recuperação anestésica ${ }^{4}$. No entanto, cabe ressaltar que o índice de Aldrete e Kroulik avalia cinco parâmetros isoladamente, o que não deve substituir a avaliação criteriosa do profissional ${ }^{10}$, sobretudo por não contemplar, de modo sistematizado, a avaliação de outros dados clínicos, como temperatura e dor.

Nessa perspectiva, os acadêmicos relataram o controle da dor aguda pós-operatória reconhecidamente como um sintoma que predispõe o paciente a eventos graves. Para isso, é preciso implementar o uso de escalas para reduzir a subjetividade da avaliação pelos profissionais e incluí-lo como um dos parâmetros a serem considerados para a alta da SRPA ${ }^{4}$. Todavia, pela subjetividade da avaliação, há evidências científicas que identificaram que a dor, para o enfermeiro, é tratada apenas como um sintoma, sendo negligenciada a classificação desta como o quinto sinal vital. Além disso, indicam que os profissionais saem despreparados da graduação para avaliar a subjetividade da dor ${ }^{11}$, circunstância que explica, em parte, a incipiência do relato do uso de escalas para avaliação da dor pelos acadêmicos participantes da presente pesquisa.

Outro dado encontrado nos depoimentos dos acadêmicos está relacionado ao cuidado de enfermagem no que se refere à manutenção da temperatura corporal. A administração de sedativos e anestésicos interfere na capacidade do hipotálamo de regular a temperatura central, ocasionando hipotermia indesejada durante o intraoperatório e na recuperação pós-anestésica ${ }^{12}$. Somam-se a isso outros fatores, como a temperatura da sala de operação, a infusão de soluções frias e a idade, que causam instabilidade de termorregulação do organismo ${ }^{11}$.

A hipotermia perioperatória está associada a vários eventos adversos, incluindo aumento do desconforto pós-operatório, do tempo de recuperação pós-anestésica, de complicações cardíacas, de quadros hemorrágicos, de infecção de sítio cirúrgico, entre outros ${ }^{12}$. A estratificação de risco de hipotermia pelo enfermeiro desde o pré-operatório contribuirá para a segurança do paciente ${ }^{13}$. Assim, a avaliação do risco e a adoção dos métodos de aquecimento precisam ser enfatizados durante o processo de formação profissional, visando ao cuidado seguro, especialmente na fase de recuperação anestésica.

Na presente pesquisa, pelos discursos de alguns acadêmicos, pôde-se entender que, das seis metas internacionais de segurança do paciente, estabelecidas pela OMS e preconizadas pela
Joint Commission International, somente três foram mencionadas pelos acadêmicos: comunicação efetiva, redução do risco de infecções associadas aos cuidados em saúde e prevenção de danos decorrentes de quedas ${ }^{14}$. Uma possível justificativa para esse achado concentra-se no fato de que o ensino sobre segurança do paciente, na graduação em saúde, persiste fragmentado, carecendo de aprofundamento e amplitude conceitual ${ }^{15}$. Além disso, enfermeiros podem não considerar o quanto suas práticas rotineiras contribuem para gerenciar os riscos e manter os pacientes seguros ${ }^{16}$. Infere-se que essa percepção pode estar vigente desde o período de formação, o que, por sua vez, dificulta o entendimento dos acadêmicos em associar suas ações/seus cuidados às metas de segurança reconhecidas mundialmente. Dessa forma, há a necessidade de aprofundar a temática durante o processo formativo dos acadêmicos de enfermagem, incluindo os participantes da presente pesquisa.

Sabe-se que a lista de verificação de segurança cirúrgica, elaborada pela OMS, tem por objetivos apoiar o uso de práticas seguras e promover a comunicação entre as equipes para reduzir e mitigar erros cirúrgicos ${ }^{1}$. Apesar de o preenchimento do checklist ocorrer em sua totalidade na sala operatória, salienta-se que sua efetiva utilização, desde antes da indução anestésica, reflete, positivamente, na evolução do paciente e na transição do cuidado para o pós-operatório. Por outro lado, observa-se que a comunicação de erros cirúrgicos, cuja finalidade é elencar ações para evitar a recorrência de casos, permanece fragilizada na perspectiva da equipe de saúde, mesmo após a implantação do protocolo de cirurgias seguras ${ }^{17}, \mathrm{o}$ que evidencia a importância de promover a comunicação e a cultura de segurança do paciente na formação acadêmica.

A comunicação efetiva está relacionada à proteção de erros $^{1}$, além de ser uma das habilidades requeridas para o exercício profissional do enfermeiro ${ }^{18}$. Dada a sua relevância, pesquisadores norte-americanos desenvolveram uma teoria de médio alcance para melhorias da comunicação na cultura de segurança do paciente ${ }^{16}$ que pode nortear o processo de ensino e aprendizagem para melhorias contínuas do processo de trabalho.

A liderança e o gerenciamento, de acordo com os participantes, auxiliam na promoção da segurança do paciente. A liderança envolve compromisso, responsabilidade, empatia, habilidade na tomada de decisões, comunicação e gerenciamento de forma efetiva e eficaz ${ }^{18}$. Ademais, evidências científicas mostram que o tipo de liderança transformacional desempenha papel ativo na criação de um clima no qual os funcionários se sentem à vontade para expressar suas preocupações de segurança, a fim de garantir e implementar práticas 
de qualidade e seguras ${ }^{19}$. Nesse sentido, salienta-se a necessidade de avançar em práticas que sustentem a formação do enfermeiro para a área gerencial, dada sua relevância para implementar ações em SRPA que visem ao cuidado seguro.

Entre os recursos necessários ao cuidado do paciente em recuperação cirúrgica e anestésica, os acadêmicos de enfermagem citaram que, além da provisão e do gerenciamento de recursos humanos, cabe ao profissional enfermeiro planejar e prescrever ações durante a permanência do paciente na SRPA, com a aplicação do processo de enfermagem, o qual constitui etapa para operacionalizar a sistematização da assistência de enfermagem, que norteia a continuidade da assistência e a qualidade do cuidado de enfermagem ${ }^{7}$. No ambiente cirúrgico, uma acadêmica de enfermagem destacou a SAEP como uma das atividades do enfermeiro na SRPA ${ }^{9}$, corroborando os achados da presente pesquisa.

Para aprimorar e auxiliar acadêmicos durante os estágios curriculares, pesquisadores nacionais elaboraram e validaram um instrumento para o registro da SAEP incluindo itens específicos para SRPA ${ }^{20}$. Acredita-se que práticas inovadoras poderão contribuir com a promoção do cuidado aos pacientes em recuperação anestésico-cirúrgica, bem como aprimorar o processo formativo de futuros profissionais enfermeiros, com foco na qualidade e na segurança do paciente em SRPA.

\section{CONSIDERAÇÕES FINAIS}

Os acadêmicos de enfermagem reconheceram ações necessárias para realizar o cuidado de enfermagem com segurança na SRPA, entre elas: verificação de sinais vitais; monitoramento de sinais e sintomas; aplicação da escala de Aldrete e Kroulik; avaliação do risco de queda e agitação; e aplicação do processo de enfermagem.

Considera-se como limitação da pesquisa a incipiência de estudos conduzidos com acadêmicos de enfermagem sobre SRPA para discussão dos resultados encontrados. Esta pesquisa poderá colaborar para o ensino em segurança do paciente em SRPA, contribuindo, assim, com a prática profissional do enfermeiro.

\section{REFERÊNCIAS}

1. World Health Organization (WHO). The second global patient safety challenge: safe surgery saves lives [Internet]. Genebra: WHO; 2009 [acessado em 22 jan. 2019]. Disponível em: http://www.who.int/patientsafety/ safesurgery/knowledge_base/SSSL_Brochure_finalJun08.pdf

2. Luckowski A. Safety priorities in the PACU. Nursing [Internet]. 2019 [acessado em 28 jul. 2019];49(4):62-5. Disponível em: https://doi. org/10.1097/01.NURSE.0000554246.74635.e0

3. Neily J, Silla ES, Sum-Ping SJT, Reedy R, Paull DE, Mazzia L, et al. Anesthesia adverse events voluntarily reported in the veterans' health administration and lessons learned. Anesth Analg [Internet]. 2018 [acessado em 12 jan. 2019];126(2):471-7. Disponível em: https://doi. org/10.1213/ANE.0000000000002149

4. Dill MCP, ArboitEL, Kaefer CT, Arboit J. Perceptions about an instrumentused for patients' evaluation and discharge from post-anesthesia care units. Rev Fund Care Online [Internet]. 2018 [acessado em 10 jan. 2019];10(3):711-9. Disponivel em: http://dx.doi.org/10.9789/2175-5361.2018.v10i3.711-719

5. Bonetti AEB, Girardello DTF, Coneglian ALA, Egevardt D, Batista J, Cruz EDA. Assistência da equipe de enfermagem ao paciente em sala de recuperação pós-anestésica. Rev Enferm UFSM [Internet]. 2017 [acessado em 11 jan. 2019];7(2):193-205. Disponível em: http:// dx.doi.org/10.5902/2179769226840

6. Ribeiro MB, Peniche ACG, Silva SCF. Complications in the postanesthesia care unit, risk factors and nursing interventions: an integrative review. Rev SOBECC [Internet]. 2017 [acessado em 10 jan. 2019];22(4):218-29. Disponível em: https://doi.org/10.5327/ Z1414-4425201700040007

7. Ribeiro E, Ferraz KMC, Duran ECM. Atitudes dos enfermeiros de centro cirúrgico diante da sistematização da assistência de enfermagem perioperatória. Rev SOBECC [Internet]. 2017 [acessado em 28 jul. 2019];22(4):201-7. Disponível em: https://doi.org/10.5327/ Z1414-4425201700040005

8. Bardin L. Análise de conteúdo. Lisboa: Edições 70; 2011.

9. Santos PB, Boezzio TF, Stamm B. Vivência acadêmica em sala de recuperação pós-anestésica: um relato de experiência. Rev Espaço Ciênc Saúde [Internet]. 2016 [acessado em 11 jan. 2019];4(1):116-23. Disponível em: http://www.revistaeletronica.unicruz.edu.br/index. php/enfermagem/article/view/5255

10. Castro FSF, Peniche ACG, Mendoza IYQ, Couto AT. Body temperature, Aldrete-Kroulik index, and patient discharge from the post-anesthetic recovery unit. Rev Esc Enferm USP [Internet]. 2012 [acessado em 31 jan. 2019];46(4):870-4. Disponível em: http://dx.doi.org/10.1590/ S0080-62342012000400013

11. Queiróz DTG, Carvalho MAC, Carvalho GDA, Santos SR, Moreira AS, Silveira MFA. 5th vital sign: nurses' knowledge. J Nursing UFPE [Internet]. 2015 [acessado em 7 nov. 2018];9(4):7186-92. Disponível em: https://doi.org/10.5205/1981-8963-v9i4a10477p7186-7192-2015 
12. Zaza KJ, Hopf HW. Thermoregulation: normal physiology, anesthetic effects, and perioperative considerations. In: Hemmings Jr. HC, Egan TD, editores. Pharmacology and Physiology for Anesthesia [Internet]. 2a ed. Rio de Janeiro: Elsevier; 2019 [acessado em 30 jan. 2019]. p. 300-10. Disponível em: https://doi.org/10.1016/ B978-0-323-48110-6.00015-6

13. Ribeiro E, Navarro NT, Armede VCB, Rodrigues HS, Valle JP, Duran ECM. Unintentional hypothermia frequency in the perioperative period of elective surgeries. Rev SOBBEC [Internet]. 2016 [acessado em 12 jan. 2019];21(2):68-74. Disponível em: https://doi.org/10.5327/ Z1414-442520160002000

14. Joint Commission International. International Patient Safety Goals [Internet]. Estados Unidos: JCl; 2019 [acessado em 30 jan. 2019]. Disponivel em: https://www.jointcommissioninternational.org/ improve/international-patient-safety-goals/

15. Bohomol E, Freitas MAO, Cunha ICKO. Patient safety teaching in undergraduate health programs: reflections on knowledge and practice. Interface [Internet]. 2016 [acessado em 15 jan. 2019];20(58):727-41. Disponível em: http://dx.doi.org/10.1590/1807-57622015.0699

16. Groves PS, Bunch JL. Priming patient safety: a middle-range theory of safety goal priming via safety culture communication. Nurs Inq
[Internet]. 2018 [acessado em 31 jan. 2019];25(4);e12246. Disponível em: https://doi.org/10.1111/nin.12246

17. Batista J, Cruz EDA, Alpendre FT, Paixão DPSS, Gaspari AP, Maurício AB. Safety culture and communication about surgical errors from the perspective of the health team. Rev Gaúcha Enferm [Internet]. 2019 [acessado em 31 jan. 2019 ];40(Esp.):e20180192. Disponível em: http://dx.doi.org/10.1590/1983-1447.2019.20180192

18. Brasil. Ministério da Educação. Parecer CNE/CES n 1133, de 7 agosto de 2001. Diretrizes curriculares nacionais dos cursos de graduação em enfermagem, medicina e nutrição. Diário Oficial da União. 2001.

19. McFadden KL, Stock GN, Gowen CR. Leadership, safety climate, and continuous quality improvement: impact on process quality and patient safety. Health care Manage Rev [Internet]. 2015 [acessado em 23 dez. 2018];40(1):24-34. Disponível em: https://doi.org/10.1097/ HMR.0000000000000006

20. Monteiro EL, Melo CL, Amaral TLM, Prado PR. Cirurgias seguras: elaboração de um instrumento de enfermagem perioperatória. Rev SOBECC [Internet]. 2014 [acessado em 12 jan. 2019];19(2):99-109. Disponível em: https://revista.sobecc.org.br/sobecc/article/ view/65 